\title{
Die Komposition personennaher Dienstleistungen von morgen
}

\author{
Christoph Lattemann $(\mathbb{D} \cdot$ Susanne Robra-Bissantz $\cdot$ Christoph Ziegler
}

Eingegangen: 19. Mai 2020 / Angenommen: 19. Juni 2020 / Online publiziert: 6. Juli 2020

(C) Der/die Autor(en) 2020

Zusammenfassung Nach den anhaltenden Erfolgen der Digitalisierung in der Industrie und im Sektor industrienaher Dienstleistungen in den vergangenen Jahren, die unter dem Schlagwort der Industrie $4.0 \mathrm{zu}$ verzeichnen sind, erfolgt nun eine Verlagerung der Schwerpunktsetzung auf die Digitalisierung personennaher Dienstleistungen (PDL). PDL zeichnen sich dadurch aus, dass sie Werte am Menschen und/oder für den Menschen schaffen. Sie kommen in allen Bereichen und Sektoren vor, in denen menschliche Interaktionen stattfinden, also nicht nur im privaten oder sozialen Bereichen, sondern auch im unternehmerischen Kontext. PDL müssen grundsätzlich an den menschlichen Bedürfnissen und Erfahrungen von Nutzern ausgerichtet sein. Dies unterscheidet sie von industriellen Produkten und Dienstleistungen, deren Entwicklung häufig durch techno-ökonomische Kriterien getrieben ist. Deswegen sind die Entwicklung und Digitalisierung von PDL auch nicht mit den bekannten Innovationsmethoden und dem grundsätzlichen Wertschöpfungsverständnis aus der Industrie 4.0 und auch nicht mit dem klassischen Konsumgütermarketing vereinbar.

In diesem Grundlagenartikel zeigen wir die Entwicklungstendenzen von PDL in Zeiten der Digitalisierung auf. Exemplarisch beleuchten wir einige, durch Megatrends getriebene und durch die Corona-Krise verstärkte, gesellschaftliche Veränderungen und daraus resultierende Konsum- und Lebensstile, die wiederum die

\footnotetext{
C. Lattemann $(\bowtie)$

Jacobs University Bremen, Campus Ring 1, 28759 Bremen, Deutschland

E-Mail: c.lattemann@jacobs-university.de

University of Agder (UiA), Agder, Norwegen

S. Robra-Bissantz

Technische Universität Braunschweig, Mühlenpfordtstraße 23, 38106 Braunschweig, Deutschland

C. Ziegler

Projektträger Karlsruhe (PTKA), Karlsruher Institut für Technologie (KIT),

Hermann-von-Helmholtz-Platz 1, 76344 Eggenstein-Leopoldshafen, Deutschland
} 
Bedürfnisse nach PDL beeinflussen. Die sich hieraus ergebenden Konsequenzen für Unternehmen werden dargestellt.

Aus den Charakteristika PDL und den theoretischen Überlegungen der Servicedominierten Logik leiten wir sieben pragmatische Prinzipien für einen notwendigen Wandel in der unternehmerischen Grundausrichtung ab und beschreiben beispielhaft eine Innovationsmethode, sowie eine Methode zur Entwicklung von Geschäftsmodellen, die eine ganzheitliche Komposition von PDL reflektiert.

Dieser Artikel bietet Praktikern Leitplanken zur Navigation durch ein neues Terrain der PDL. Für die Wissenschaft wollen wir mit diesem Artikel die Diskussion über die Entwicklung digital-unterstützter PDL eröffnen.

Schlüsselwörter Personennahe Dienstleistungen · Service-dominierte Logik · Mindset · Geschäftsmodelle · Innovationsmodelle

\section{The Composition of Personal Services of Tomorrow}

Abstract Over the past 10 years, the industry experienced an ongoing and great technology and digitalization boost, promoted under the concept of "Industry 4.0". This development includes not only the industrial production but as well related services. Now, we are at the verge of a consecutive era, the digitalization of personal services. Personal services are characterized by a value creation for people and with people. These services exist in all sectors where personal interactions take place: in the private and social sector, as well as in a corporate context.

Personal services must focus on user needs and experiences. In this aspect they fundamentally differ from industrial products and services, which focus on technoeconomic aspects. That is why existing strategies, business models and innovation models from the industrial or consumer goods markets cannot be used for developing and providing personal services.

This article will describe the developmental path of personal services in times of digitalization, and megatrends such as connectivity, security, health and individualization - which are fuelled by the corona crisis. The digitalization and the mentioned megatrends result in social changes and new consumer- and life-styles, which in turn change the needs for personal services. The resulting consequences for companies will be presented.

We will present seven pragmatic principles for the necessary change in a corporate mind-set derived from the characteristics of personal services and based on theoretical considerations of the service dominant logic. Further, we describe an innovation method and a business canvas which adequately address the particularities of personal services in times of digitalization.

Keywords Personal Services · Service Dominant Logic · Mind-set · Business Model · Innovation Model 


\section{Einführung}

Durch die Folgen der Corona-Pandemie ordnen sich die Relevanzen von Megatrends (Zukunftsinstitut 2020) als Entwicklungskonstanten der globalen Gesellschaft neu. Gerade die Megatrends, die maßgeblich die Entwicklung von Dienstleistungen beeinflussen, wie Konnektivität, Sicherheit, Gesundheit und Individualisierung, werden durch Digitalisierung und Corona-Krise noch forciert (Gatterer 2020). Es verändern sich Lebensmuster, Konsumstile, Nutzerbedürfnisse, sowie Arbeitsorganisationen und -prozesse, was wiederum Auswirkungen auf die Anforderungen an Dienstleistungen hat (u. a. Hipp 2013; BMBF 2016). So bedingt zum Beispiel die Digitalisierung das Medienverhalten von Nutzern. Das Medienverhalten der Nutzer verändert wiederum das Angebot an und die Erstellung von Dienstleistungen.

Vor allem ist es in dieser sich verändernden, digitalisierten Welt Nutzern nun möglich, Dienstleistungen selbst zu gestalten, selbst zu bestimmen, welche Dienstleistungen sie in Anspruch nehmen wollen und welche Werte die Dienstleistung für sie erbringen sollen. Deswegen muss eine erfolgreiche Dienstleistung konsequent von der Nutzerperspektive aus gedacht werden. Damit ist das alte, angebotsorientierte Spiel in der Dienstleistungserbringung vorüber. Ein neues, nutzerzentriertes Spiel hat begonnen. Diese Veränderungen verlangen einen Wandel des grundlegenden Verständnisses (Mindset) der Wertschöpfung, indem von einer angebotsorientierten Unternehmensphilosophie Abstand genommen und eine nutzerzentrierte, ko-kreative Dienstleistungsperspektive eingenommen wird. Dieser Wandel umfasst gleichermaßen die Bestimmung von Ökosystemen, Geschäftsmodellen und Innovationskonzepten. Dies gilt insbesondere für personennahe Dienstleistungen (PDL).

PDL sind typischerweise im Hotel- oder Gaststättengewerbe, in Kindergärten, in Bildungseinrichtungen, in der Pflege, bei der Haushaltshilfe, in der Quartiersentwicklung, im Handwerk, oder auf Verkaufsplattformen zu finden. Sie kommen aber auch grundsätzlich in allen Sektoren vor, in denen menschliche Interaktionen stattfinden, also nicht nur im privaten oder sozialen Bereichen, sondern auch im unternehmerischen Kontext. Sie definieren sich darüber, dass sie Werte für und am Menschen schaffen, indem der Mensch selbst im Mittelpunkt eines interaktiven Wertschöpfungsprozesses steht (Lattemann et al. 2019). Dies grenzt PDL von anderen Dienstleistungen ab, wie zum Beispiel Reparaturen oder Wartungen von Maschinen oder standardisierte Transaktionen im Zahlungsverkehr.

Ziel dieses Grundlagenartikels ist es, zum einen die Bedeutung von PDL für Gesellschaft und Wirtschaft in Zeiten sich ändernder Umweltbedingungen (zunehmende Digitalisierung, Corona-Krise, Verschiebungen von Megatrends) und somit die Relevanz des Themas aufzeigen. Zum anderen wollen wir akademische und praktische Aspekte von PDL anreißen und somit einen breiten Diskurs anstoßen.

Hierzu werden im Kap. 2 die Eigenschaften von PDL beschrieben, die Rolle der Digitalisierung für PDL aufgezeigt, sowie spezielle Anforderungen der PDL an Service-Ökosysteme dargestellt. Die sich hieraus ergebenden Konsequenzen für Unternehmen werden herausgearbeitet. Kap. 3 stellt dar, warum Unternehmen bei der Erbringung digitaler PDL einen Paradigmenwechsel in ihren Zielkoordinaten vollziehen müssen und auf welchen Grundpfeilern dieser Wandel fußt. Hierfür wird die Service-dominierte Logik (SDL) (Vargo und Lusch 2016) vorgestellt. Für die 
konkrete Umsetzung dieses Paradigmas in eine geänderte Unternehmenspraxis werden sieben pragmatische Prinzipien abgeleitet. Diese Prinzipien zeigen auf, dass es für Unternehmen zukünftig kein einfaches ,weiter so“ gibt, sondern neue Innovationskonzepte und Geschäftsmodelle eingeführt werden müssen. Hierzu werden in Kap. 4 Innovationskonzepte aus der Industrie und dem Sektor der industrienahen Dienstleistung kurz vorgestellt, die bereits Elemente auf dem Weg zu einer ServiceOrientierung aufzeigen. Darauf folgend wird ein für die Entwicklung von digitalisierten PDL adäquates, agiles und kundenzentriertes Innovationskonzept und deren zentrale Elemente dargestellt, sowie ein Werkzeug (Canvas) für die Gestaltung von kundenorientierten Geschäftsmodellen für PDL exemplarisch präsentiert. Der Beitrag schließt mit einem zusammenfassenden Ausblick.

\section{Personennahe Dienstleistungen in der Digitalen Transformation}

PDL gelten als Beschäftigungsmotor, schaffen wirtschaftliche Leistungsfähigkeit, zukunftssichere Arbeitsplätze, soziale Gerechtigkeit, Lebensqualität und bilden auch häufig die Basis für ein soziales Miteinander (Bedien.org 2020). Eine PDL ist darüber definiert, dass sie ihre Wirkung besonders nah am Menschen, beziehungsweise am Nutzer entfaltet, sei es als Beschäftigter, Kunde, Patient oder Klient. Bei PDL geht es um die Erfüllung von individuellen, menschlichen Bedürfnissen in der konkreten Nutzung, dem sogenannten Value-in-Use (Grönroos und Voima 2013) und der Gestaltung von Erfahrungen für den Nutzer in der Interaktion, dem Value-in-Interaction (Geiger et al. 2020). Die Werteerstellung baut auf Kommunikation, Interaktion und Kollaboration der beteiligten Wertschöpfungspartner und den Austausch ihrer komplementären Ressourcen auf. In Abgrenzungen zu anderen Dienstleistungen steht also bei PDL der Mensch als Nutzer, mit seinen individuellen, menschlichen Bedürfnissen, im Mittelpunkt eines interaktiven Wertschöpfungsprozesses. Aus der Grundüberlegung des Value-in-Use ergibt sich darüber hinaus, dass der Wert erst durch den Nutzer selbst geschaffen wird. In dieser Logik entwickeln die an der Wertschöpfung beteiligten Akteure lediglich ein Wertversprechen für den Nutzer (Grönroos, Voima 2013).

Praktische Beispiele und Forschungsvorhaben (siehe auch die Beiträge im vorliegenden HMD Themenheft) zeigen, dass eine sinnhafte Automatisierung zum Beispiel über digitale Plattformen, mobile Applikationen für Smartphones, KI, AR oder Cloudcomputing zu neuen, nutzerzentrierten und individualisierten PDL führen kann (Robra-Bissantz 2018). In diesem Kontext werden im Folgenden Einflüsse der zunehmenden Digitalisierung auf PDL dargestellt (Abschn. 2.1) und die Anforderungen an die Entwicklung und Gestaltung von PDL und deren Ökosysteme und Geschäftsmodelle (Abschn. 2.2) aufgezeigt.

\subsection{Digitalisierung und PDL - ein iteratives Zusammenspiel}

In den Sektoren der industrienahen Dienstleistungen, die einem hohen Effizienzdruck unterliegen oder deren Dienstleistungen zum großen Teil auf Informationen fußen, wie zum Beispiel im Finanzsektor, in der Produktion, in der Logistik oder 
in der Telekommunikation, sind durch die Digitalisierung viele innovative und zum Teil hochgradig individualisierte Dienstleistungen entstanden. In Sektoren, die stärker am Menschen orientiert sind, wie zum Beispiel im Hotel- oder Gaststättengewerbe, in Kindergärten, in Bildungseinrichtungen ist das Potential zur Gestaltung neuer, erfahrungsbasierter Dienstleistungen jedoch noch lange nicht ausgeschöpft.

Die Digitalisierung verläuft in diesem Sektor auch nicht linear, sondern in Iterationen, in denen sich technologische Entwicklungen, Kundenpräferenzen und veränderte Lebensstile gegenseitig bedingen. Da dieser Prozess nicht planbar ist, benötigt dieser Wandel Zeit, weil sich die Komponenten im System immer wieder anpassen müssen, mit all den Konsequenzen für ein gesellschaftliches Miteinander und auf allen Ebenen der Wirtschaft.

Diese iterative Entwicklung zeigt sich auch in der graduellen Digitalisierung der Wirtschaft in den vergangenen Jahren. In Etappen ist ein Perspektivwechsel bei Unternehmen zu beobachten; von der Fokussierung auf Produkte und Technologien über Produkt-Dienstleistungsbündel bis zu PDL. Mit diesen Etappen ist implizit ein stufenweiser Wandel in der unternehmerischen Geisteshaltung eingegangen, der sich in den jeweiligen Geschäftsmodellen, Innovationskonzepten und organisationalen Strukturen widerspiegelt.

Dabei hatte die Digitalisierung ihren Durchbruch in der industriellen Produktion (Industrie 4.0) mit der Implementierung intelligenter und digitaler vernetzter Systeme. Bei dieser Transformation liegt der Fokus primär auf der techno-ökonomisch getriebenen Automatisierung von und Effizienzsteigerung in Wertschöpfungsprozessen in der Entwicklung, Fertigung, Nutzung und Wartung von Produkten sowie im Informationsaustausch zwischen Maschinen, beziehungsweise zwischen Mensch und Maschine (Zollenkop und Lässig 2017).

Die zweite Digitalisierungsetappe, in der wir uns derzeit befinden, umfasst die Digitalisierung von Produkt-Dienstleistungssystemen zu sogenannten Smart Services in der industriellen und industrienahen Produktion (Beverungen et al. 2017). Innovative industrienahe Dienstleistungen zeichnen sich dadurch aus, dass sie kundenund bedarfsorientiert sind und hierfür die zunehmende digitale Vernetzung zwischen Produkten und zwischen verschiedenen Akteuren des Service-Ökosystems ausnutzen. Hieraus entstehen Nutzen für Kunden beispielsweise in Form der Vermeidung von unerwünschten und teuren Stillstandzeiten durch eine vorausschauende Überwachung und Wartung von Produktionsanlagen (Pöppelbuß und Durst 2017). Auch hier liegt der Fokus der Entwicklung primär auf einer techno-ökonomischen Automatisierung und Effizienzsteigerung und nicht auf der Befriedigung menschlicher Bedürfnisse.

In einem dritten Schritt, der den industriellen Entwicklungen nun mit Verzögerung folgt und durch die Corona-Pandemie deutlich an Fahrt gewinnt, liegt der Fokus auf der Digitalisierung von PDL. Die Orientierung weg vom Produkt, hin zum Menschen führt zu speziellen Anforderungen an die Digitalisierung. Es geht nicht mehr nur um die Integration von intelligenten Systemen und Technologien, um die Unterstützung von operativen Tätigkeiten oder um die Integration von Anspruchsgruppen in die Wertschöpfungskette über effiziente und individuelle Kundenschnittstellen. Auch spielen die klassischen Kennzahlen aus der industriellen Produktion wie Kosteneffizienz, Durchlauf- und Wartungszeiten, oder produzierte 
Stückzahl pro Zeiteinheit für die Erstellung von PDL nur noch eine untergeordnete Rolle. Vielmehr geht es bei PDL um die Erfüllung des bereits oben erwähnten Value-in-Use (Grönroos und Voima 2013) und dem Value-in-Interaction (Geiger et al. 2020), wobei der Value auf ein menschliches Bedürfnis abzielt. Entsprechend müssen digitale PDL gleichermaßen nutzerzentrierte, technische sowie ökonomische Gestaltungselemente integrieren. Dies bedeutet eine Abkehr von einem getrennten Denken in Prozessinnovationen, funktionalen oder emotionalen Innovationen, hin zu umfassenden Erfahrungsinnovationen (Matthews und Wringley 2011). Diese multiperspektivische Verknüpfung von Themen führt in der praktischen Umsetzung sowie in der Forschung zu digitalen PDL zu konkreten Herausforderungen.

Innovative Unternehmen zeigen bereits heute, wie mit der konsequenten Ausrichtung am Kundennutzen und -wert statt am Produkt, mit der aktiven Partizipation und Integration von Kunden in der Wertschöpfung (Lattemann und Robra-Bissantz 2006) und mit neuen Interaktions- und Kooperationsmechanismen in innovativen Service-Ökosystemen strategische Wettbewerbsvorteile generiert werden können. In der Musik- und Videobranche sind eindrucksvolle Beispiel Spotify, Apple und TikTok. Darüber hinaus entwickeln sich in anderen Bereichen der PDL Sharing Economy-Konzepte, wie zum Beispiel von Airbnb, Uber oder Youtube, und schaffen individuelle, ko-kreative Angebote für und mit Kunden. In viele weitere, auch soziale Bereiche ist diese nutzerorientierte Sichtweise übertragbar. Gerade in der aktuellen Corona-Krise zeigen Projekte auf, wie sich beispielsweise ältere oder freiwillig arbeitende Menschen mit Hilfe digitaler Medien direkt in eine Dienstleistung einbringen können (Hofmann 2020). Hier zeigt sich, dass Mitarbeiter zu Kunden und Kunden zu Mitarbeitern werden, die werthaltige Dienstleistungen erbringen. Dies fordert neue Formen der Zusammenarbeit in adäquat gestalteten Service-Ökosystemen.

\section{2 Ökosysteme für PDL - fein abgestimmte Kompositionen in der Gesellschaft}

Es zeigt sich, dass bei der Erstellung von Dienstleistungen immer verschiedenste Akteure mit ihren komplementären Ressourcen beteiligt sind. Systeme, Menschen, Prozesse, Produkte, Dienstleistungen und Technologien müssen ineinandergreifen und ganzheitlich miteinander abgestimmt und verknüpft werden. Hieraus bildet sich ein Service-Ökosystem. Wesentlicher Bestandteil eines solchen Ökosystems ist es, dass sich alle Entscheidungen und Tätigkeiten in aller Konsequenz an der Werteerstellung für Endnutzer orientieren. Diese Nutzerzentrierung ist zwar kein neuer Gedanke, aber gleichwohl noch lange kein common sense bei traditionellen Dienstleistern. Ein recht neuer Gedanke ist es hingegen, den Endnutzer als integrativen Teil des Ökosystems, zugleich als Adressat und gleichberechtigten Wertschöpfungspartner, zu verstehen. Hierbei geht die Idee des gleichberechtigten Wertschöpfungspartners viel weiter als der „Prosumer"-Gedanke, weil sich die Dichotomie zwischen Produzent und Konsument in der Sichtweise der SDL schlichtweg aufhebt (Vargo und Lusch 2011). Entsprechend werden in einem Service-Ökosystem nicht nur Werte für den Endnutzer geschaffen, sondern jeder Wertschöpfungspartner stellt gleichermaßen eine Ressourcenquelle dar und repräsentiert einen Nachfrager nach Werten, seien diese materieller oder immaterieller Natur. Da nun die Nutzer PDL integra- 
le Bestandteile von Ökosystemen sind, stehen in einem Ökosystem wahrscheinlich nicht mehr alleinig wirtschaftlich-performante Elemente im Vordergrund, sondern auch gesellschaftliche Aspekte, Konnektivität und sicherheitsorientierte Konzepte (siehe Megatrends) werden zunehmend Rollen spielen.

Dies sind nun aber alles keine Kriterien, die ein Service-Ökosystem von einem PDL-Ökosystem abgrenzen. Spezifisch für ein Ökosystem für PDL ist vor allem das iterative Zusammenspiel zwischen einer dynamischen Umwelt (einschließlich zunehmender Digitalisierung), veränderten, menschlichen Bedürfnissen und Verhaltensweisen der Nutzer, sowie der Gestaltung der PDL. Dies zeigt sich beispielhaft gerade in der aktuellen Situation. Derzeit verunsichert die Corona-Krise viele Menschen. Der Megatrend des Strebens nach Sicherheit verstärkt sich. Dieses kollektive Empfinden wird sich zukünftig wahrscheinlich als grundlegendes Bedürfnis und verstärkt in Nutzerpräfenzen für PDL zeigen und zwangsläufig auf die Technologieentwicklung auf allen Ebenen der Gesellschaft, sowie auf die Ausgestaltung der PDL selbst auswirken.

Diese Dynamiken erfordern eine besonders feine Komposition eines Ökosystems unter agilen Bedingungen, für die es momentan noch keine angemessenen Gestaltungsansätze gibt. Ebenso wichtig ist auch die Bestimmung von Entwicklungsstrategien und -methoden für PDL und eine Sicherstellung der Gewinnerbringung durch erfolgsversprechende Geschäftsmodelle und Design Konzepte. Entsprechend muss die Digitalisierung im Kontext PDL grundlegend und übergreifend gedacht, implementiert und evaluiert werden.

\section{Digitalisierung und PDL - eine neue Geschäftslogik}

Warum ist ein Wechsel der zugrundeliegenden Geschäftslogik in Unternehmen in der Entwicklung und Bereitstellung von PDL notwendig? Die Antwort auf die Frage liegt auf der Hand. Produkte und Dienstleistungen, auch jene, die zum Beispiel im Kontext von Industrie 4.0 stehen, haben ihren Ursprung immer seltener in einer neuen Technologie oder einer Software-Idee. Sie beziehen ihre Quelle aus einem Grundverständnis über die Bedürfnisse des Menschen zum Beispiel hinsichtlich Gesundheit, Sicherheit, Individualität, Konnektivität - die sich auch in den Megatrends spiegeln. Entsprechend werden auch Aspekte wie die (digital) Customer Experience (Robra-Bissantz und Lattemann 2019), das Dienstleistungsdesign und GamificationAnsätze (Stieglitz et al. 2017) zu zentralen Elementen für Nutzer - Aspekte, die in industrienahen Dienstleistungen in der Regel keine oder nur eine untergeordnete Rolle spielen. PDL sind nutzer-zentriert und zielen auf die Bedürfnisbefriedigung von Menschen ab. Also müssen Lösungen im Bereich der PDL konsequent auf die Nutzerperspektive ausgerichtet und auch aus dieser Perspektive gedacht werden.

Eine einfache Geschäftslogik bei der Entwicklung von Lösungen für Nutzer muss dementsprechend Fragen in der folgenden Reihenfolge beantworten:

1. Was sind die Werte und Bedarfe der Nutzer?

2. Welche beteiligten Netzwerkpartner können und sollen mit ihren Ressourcen an der Werteerstellung teilnehmen? 
3. Welche Dienstleistung kann für die Erfüllung der Bedarfe zur Verfügung gestellt werden und welche Rolle spielt die Digitalisierung?

4. Welches Werteversprechen kann das Unternehmen selbst für die Erstellung des Kundenwertes beitragen?

5. Welchen Wert möchte das Unternehmen für sich aus der Dienstleistung ziehen?

Diese Logik ist ein Gegenentwurf zum traditionellen Produkt-orientierten, unternehmerischen Denken (der sogenannten Goods-Dominant Logic - GDL), bei dem der Wertschöpfungsgedanke beim Produkt anfängt und geschaut wird, welche Ressourcen ein Unternehmen selbst hat, um eine Leistung für einen Kunden zu erbringen.

Es scheint jedoch noch heute so zu sein, dass die GDL den Main-Stream in der Praxis, in der Forschung und in der Lehre bestimmt. Dazu mag die in den letzten Jahren zunehmende Zersplitterung der Fachgebiete beigetragen haben, die sich im Kern mit Dienstleistungen beschäftigen, wie dem Dienstleistungsmanagement, dem Marketing, dem Service-Engineering, dem Service Design, dem Interaction Design, oder der Human-Computer-Interaction. Dass sich in der Vergangenheit kein gemeinsames Dienstleistungsverständnis entwickelt hat, liegt wohl vor allem auch daran, dass die genannten Wissensbereiche in ihrer praktischen Anwendung auf unterschiedliche Forschungsmethoden, -ansätze und -objekte zurückgreifen und keine einheitlichen Terminologien verwenden. Somit ist ein einheitliches Verständnis nur schwer zu entwickeln, aber es zeichnet sich Bewegung ab. Technik, Wirtschaft, Design und Nutzererfahrungen werden zunehmend mehr zusammengedacht und in vielen der Fachbereiche ist mittlerweile gemein, dass sie sich nutzerzentriert ausrichten. Das Konzept der SDL (Vargo und Lusch 2016) setzt sich langsam durch. Dies führt zu der Entwicklung ganzheitlich gedachter Geschäftsmodelle und Innovationsmethoden, auch für die Entwicklung von zeitgemäßen, digitalisierten PDL.

\subsection{Die Bedeutung der Service-dominierten Logik}

Die oben dargestellte Notwendigkeit eines Perspektivwechsels in der praktischen Erbringung von PDL findet ein entsprechendes theoretisches Spiegelbild im Forschungsfeld der SDL, das - entgegen der GDL - zentral die Dienstleistung und eine Nutzer-Wert-Orientierung in den Vordergrund stellt. Aus dieser Perspektive folgt, dass Produkte Vermittler für Dienstleistungen und Dienstleistungen wiederum Vermittler von Nutzerwerten sind. PDL werden durch Prozesse definiert, die in der Interaktion zwischen Anbieter und Nutzer (Value-in-Interaction) im Moment der Inanspruchnahme durch den Nutzer entstehen (Value-in-Use). Der Kunde ist hierbei immer zentral selbst an der Wertschöpfung (Ko-Kreation) beteiligt (Lattemann und Robra-Bissantz 2006), da der Wert nur durch ihn selbst entstehen kann. Alle anderen Wertschöpfungspartner stellen lediglich Wertversprechen für den Nutzer zur Verfügung. In dieser Logik wird gleichsam die Dichotomie zwischen produzierenden, Werte aufbauenden Unternehmen und konsumierenden, Werte vernichtenden Kunden durchbrochen (Vargo und Lusch 2011).

Auch propagiert die SDL die Notwendigkeit eines Service-Ökosystems, das sich aus heterogenen Akteuren zusammensetzt, die mit intelligent gestalteten (digitalen) 
Schnittstellen (Robra-Bissantz 2018) zwischen den Wertschöpfungspartnern verbunden sind und über den Austausch von Wertbeiträgen, beziehungsweise materiellen und immateriellen Ressourcen miteinander interagieren, um gemeinsame Ziele zu erreichen (Wieland et al. 2012).

Diese neue, dienstleistungsdominierte Gedankenwelt muss in Unternehmen mit einem veränderten Verständnis (Mindset), mit neuen Innovationsmethoden und Geschäftsmodellen einhergehen. Kunden müssen als zentrale Gesprächspartner verstanden werden.

Die dargestellten Denkansätze und Arbeitsweisen in der Erbringung von PDL können durch die folgenden sieben Prinzipien beschrieben werden: (1) Nutzerzentriertheit, (2) Integration, (3) Kollaboration, (4) Agilität, (5) Ko-Kreativität, (6) Interdisziplinarität und (7) Digitalisierung. Diese sieben pragmatischen Prinzipien durchziehen die im Folgenden dargestellten Methoden zur Erstellung von Geschäftsmodellen, Entwicklungskonzepte und neue Arbeitsmethoden und -konstellationen und dienen als praktische Leitplanken für die Etablierung einer neuen Geschäftspraxis.

\subsection{Prinzipien für ein neues Mindset}

Nutzerzentriertheit/Individualisierung: Um Menschen in ihren Bedürfnissen angemessen zu bedienen, müssen zwangsläufig die Menschen, bzw. Nutzer, und ihre Bedürfnisse verstanden werden, für die eine PDL entwickelt und zur Verfügung gestellt wird. Der Nutzen einer PDL kann dabei für verschiedene Nutzer in ihren verschiedenen Situationen unterschiedlich sein. Eine PDL sollte daher immer aus der Perspektive der Nutzer, die sie in Anspruch nehmen und bereitstellen, entwickelt werden und sich an ihren menschlichen Bedürfnissen und Motivationen orientieren, um eine angenehme und erfreuliche Dienstleistungserfahrung (Customer Experience) zu generieren.

Integration: Ein einziger Dienstleister wird nicht über alle materiellen und immateriellen Ressourcen verfügen können, um eine PDL, die auf viele Nutzer in ihren speziellen Situationen ausgerichtet ist, alleine anzubieten. Eine offene, ganzheitliche Sicht auf mögliche Partner bei der Entwicklung von Ökosystemen für PDL integriert daher alle diejenigen Dienstleistungen und die daran beteiligten Menschen, Organisationen, Technologien oder Produkte, die in ihrer Gesamtheit in der Lage sind, als ineinandergreifendes System auf unterschiedliche Kontexte und Präferenzen der Kunden in ihrer Problemlösung einzugehen. Dabei kann der Kontext, indem man den Kunden anzutreffen vermutet, ein guter Ratgeber für zu integrierende Partner sein.

Kollaboration: Die Gestaltung von PDL muss alle beteiligten Akteure aktiv in die Analyse- und Entwicklungsprozesse einbeziehen. Das heißt, Lösungen werden gemeinsam mit Mitarbeitern, Entscheidungsträgern, Nutzern und anderen Stakeholdern, die an der Entstehung und Bereitstellung einer PDL beteiligt sind, entwickelt und umgesetzt. Für die Gestaltung von Beziehungen und Regeln zwischen den Stakeholdern (Governance-System) im Service-Ökosystem bietet es sich daher an, über Anreize, gegenseitigen Nutzen und gemeinsame Zielstellungen nach einer möglichst 
guten Zusammenarbeit zu streben, in welcher die Partner über die Zeit Vertrauen und Verbundenheit miteinander entwickeln.

Agilität: Der Kundennutzen einer PDL ist immer kontext- und situationsspezifisch. Die Ausrichtung an dem Kundennutzen führt so zwangsläufig zu einem agilen Vorgehen, da der Nutzer in eine dynamische Umwelt eingebunden ist. Entsprechend der Dynamik des Umfeldes verändern sich die Werte und menschlichen Bedürfnisse der Nutzer sowie die der Akteure im Ökosystem. Innovationszyklen müssen diese Dynamik reflektieren und Mechanismen vorhalten, um kurzfristig auf sie zu reagieren. Im besten Fall sind (digitale) Plattformen für die PDL-Ökosysteme direkt derart gestaltet, dass sie Veränderungen beobachten und sich als lernende Systeme daran anpassen können.

Ko-Kreativität: Der immaterielle Charakter einer PDL, der Value-in-Use und der Value-in-Interaction sind sowohl für zukünftige Nutzer als auch für die anderen Partner kaum a priori greifbar, da sie auf Erlebnisse und Erfahrungen (Customer Experiences) basieren und die eigentliche Wertschöpfung erst in der Nutzung, individuell und situationsabhängig, entsteht. Deswegen müssen in der Dienstleistungsentwicklung andere Methoden - im Vergleich zur Produktentwicklung - eingesetzt werden, um im Sinne einer gemeinsamen Kreativität zunächst, zum Beispiel über nachvollziehbare Analysen, ein gemeinsames Verständnis der Zielstellungen aller Akteure zu erreichen, um dann die Ideen für Innovationen, zum Beispiel über Visualisierung erkennbar und begreifbar zu machen.

Interdisziplinär: Die Entwicklung und Bereitstellung von PDL verlangt das Zusammenspiel vieler unterschiedlicher Tätigkeitsfelder, Fachgebiete und Kompetenzen. Daher sollten an der Entwicklung von Dienstleistungen aus den Organisationen der Partner Experten aus verschiedensten Disziplinen beteiligt sein, die zur interdisziplinären Arbeit fähig und willens sind.

Digitalisierung: Wie oben dargestellt, existiert ein iteratives, sich gegenseitig bedingendes Zusammenspiel aus technologischer Entwicklung, veränderten Nutzerpräferenzen, menschlichen Bedürfnissen und veränderten Lebensstilen. Deswegen ist es wesentlich neben der Nutzerzentrierung in der Gestaltung von PDL auch die Potenziale der Digitalisierung auf allen Ebenen zu berücksichtigen.

Die Übertragung dieser sieben Prinzipien in die unternehmerische Praxis zeigt die Unterschiede zum traditionellen, unternehmensfokussierten, produktorientierten und silodominierten Arbeitsweisen in Unternehmen auf. Die Prinzipien definieren das neue unternehmerische Mindset. Elemente dieses neuen Mindsets finden ihren Niederschlag in neueren Methoden und Prozessen der PDL-Entwicklung sowie in Methoden zur Entwicklung von Geschäftsmodellen, die im Folgenden vorgestellt werden. 


\section{Methode zur Gestaltung von PDL, Geschäftsmodell und Service Ökosystem}

Lange Zeit hat sich der Prozess der Entwicklung von Dienstleistungen an den produktorientierten und linearen Prozessen der Industrie orientiert. Dies zeigt beispielhaft der DIN-Fachbericht 75:1998 zur entwicklungsbegleitenden Normung für Dienstleistungen (Beuth 1998), in der zum Beispiel eine Kundenorientierung und ein agiles Vorgehen fehlen.

Seit dem Ende der 1990er Jahre haben sich parallel zu den Methoden der industriellen Produktion das Service Engineering (Böhmann et al. 2014) und das Smart Service Systems Engineering (Beverungen et al. 2017) (SSSE) als systematische Verfahren zur Entwicklung von digitalen Dienstleistungen etabliert. Hierbei wurde überwiegend auf Vorgehensweisen, Prozessmodelle und Methoden aus den Ingenieurswissenschaften und dem Innovationsmanagement zurückgegriffen. „Leitend waren dabei die Ideen und Verfahren der Produktgestaltung“ (Beuth 1998). Jedoch erst mit dem Konzept des SSSE hat sich die Idee der Digitalisierung bis zur zu entwickelnden Dienstleistung durchgezogen. Die Grundidee beim SSSE ist es aber immer noch, dass digitale Dienstleistungen ihre Werte durch die Erweiterung von physischen Produkten schaffen. Der immaterielle Charakter digitaler Dienstleistungen unter der Berücksichtigung veränderter Logiken in der Wertschöpfung wird in diesen Methoden entsprechend nicht reflektiert.

Ein für die Dienstleistungsinnovation passender Ansatz hat sich in den letzten Jahren mit dem Kunden-fokussierten, interdisziplinären, agilen und ko-kreativen Innovationsansatz des Service Design Thinking etabliert. Studien zu diesem Ansatz zeigen, dass die Einführung solcher agiler, ko-kreativer Verfahren in Unternehmen und die damit verbundene Transformation eines traditionell-orientierten Unternehmens hin zu den Prinzipien und Denkweisen der SDL nicht trivial ist, und dass sich das Mindset im Unternehmen mit der Nutzung dieser Verfahren ändern muss. Mitarbeiter müssen mit den neuen Arbeitsweisen, Methoden und der nutzerzentrierten Sichtweise vertraut sein, deren Notwendigkeit verstehen, und schlussendlich in täglichen Arbeitsprozessen anwenden. Dies muss mit einem organisatorischen Wandel einhergehen und benötigt Zeit (Fischer et al. 2020).

Aufgrund der Relevanz für die Erstellung von PDL wird das Service Design Thinking im Folgenden kurz vorgestellt und aufgezeigt, wie dieses Verfahren mit den sieben identifizierten Prinzipien korrespondiert.

\subsection{Service Design Thinking}

Das Service Design Thinking ist ein interdisziplinärer Ansatz, der die Prinzipien des SDL reflektiert und die sieben dargestellten Prinzipien umsetzt. Das Service Design Thinking besteht aus drei grundlegenden Elementen: dem Design Thinking Prozess, den Design Thinking Methoden und dem Design Thinking Mindset (Brenner et al. 2016). Das Service Design Thinking bedient dabei die oben abgeleiteten Prinzipien wie folgt: 
Nutzerzentriertheit: Der Design Thinking Prozess folgt der Logik eines nutzerzentrierten Designs. Der Prozess umfasst vereinfacht beschrieben drei Phasen; die nutzerzentrierte Problemerkennung, die zielgruppenspezifische Ideenentwicklung sowie die Entwicklung und Validierung eines Prototyps. In einigen Design Thinking Modellen, wie dem DETHIS Verfahren, ist zusätzlich die Entwicklung eines Geschäftsmodells Teil der prototypischen Implementierung (Redlich et al. 2019). In jeder Phase des Innovationsprozesses wird garantiert, dass der Nutzer im Zentrum der Entwicklung und des Tests der PDL steht und das Prinzip der Nutzerzentriertheit gewahrt wird.

Integration: Design Thinking wird immer in Teams mit Teilnehmern von verschiedenen Disziplinen und von verschiedenen Stakeholdern (interne und externe Stakeholder) durchgeführt. Hiermit wird zum einen eine Interdisziplinarität gewahrt und zum anderen garantiert, dass eine integrative Dienstleistung entwickelt wird.

Kollaboration \& Interdisziplinarität: Der Design Thinking Prozess zielt darauf ab, Methoden zum Beispiel der Ingenieurs-, Design-, Sozial- und Wirtschaftswissenschaften kombiniert zu nutzen, um kreative Lösungen für komplexe Probleme zu entwerfen (Johansson-Sköldberg et al. 2013). Durch den im Service Design Thinking entwickelten Mindset und durch unterstützende Methoden wird die Kollaboration zwischen den beteiligten Akteuren zur interdisziplinären Arbeit gefördert.

Agilität: Die Design Thinking Phasen werden in kurzen, zeitlichen Zyklen iterativ durchgearbeitet, womit das Prinzip der Agilität im Prozess implizit verankert ist.

Ko-Kreativität: Zur Ko-Kreativität werden im Service Design Thinking bewusst Methoden eingesetzt, die darauf abzielen, den immateriellen Charakter einer Dienstleistung, mit Value-in-Use und Value-in-Interaction, verständlich zu machen. Dies erfolgt zum Beispiel über die Darstellung von Dienstleistungsabläufen (ServiceBlueprints, Customer Journeys), -ideen und -konzepten mit Visualisierungstechniken (Rollenspiele mit Mock-Ups) und Erzähltechniken (Story-Telling).

Digitalisierung: Das Prinzip der Digitalisierung wird auf zwei Ebenen berücksichtigt. Zum einen findet im Design Thinking Prozess eine nutzerzentrierte Problemanalyse statt, die Umweltveränderungen - und somit durch Digitalisierung induzierte Verhaltensveränderungen - implizit berücksichtigt. Zum anderen können auch immer digitalisierte Lösungen mitgedacht werden.

\subsection{Methoden zur Entwicklung von Geschäftsmodellen und Service- Ökosysteme}

Wie die Ausführungen zeigen, muss die Entwicklung von PDL im Zeitalter der digitalen Transformation in Bezug zu organisationalen Strukturen, Ressourcen und Menschen grundlegend und übergreifend (neu) gedacht, implementiert und evaluiert werden. Dies spiegelt sich auch in der Darstellung von Geschäftsmodellen wider. In der Praxis gibt es eine Unzahl von Werkzeugen zur Beschreibung, Analyse und 
Entwicklung von Geschäftsmodellen. Diese Werkzeuge haben sich parallel zu den Innovationsmethoden entwickelt und beschreiben ebenso einen Pfad von einer industriellen Produktorientierung über Produkt-Dienstleistungssysteme bis zur PDL.

Die Business Model Canvas (Osterwalder et al. 2005) und die Value Proposition Canvas (Osterwalder et al. 2014) sind wohl die bekanntesten Werkzeuge zur Ableitung von Geschäftsmodellen. Beide Verfahren fokussieren auf die zu entwickelnde Value Proposition für den Kunden, die wiederum aus Unternehmenssicht definiert wird. Hierbei kommt der (individuelle) Nutzer und seine Bedürfnisse zu kurz, denn diese Modelle sehen den Kunden als Subjekt und nicht als ko-kreativen Partner in der Wertschöpfung. Vor allem sind diese Werkzeuge für die Entwicklung von Dienstleistungen wenig geeignet, weil sie den Gedanken des Service-Ökosystems nur unzureichend implizieren. Auch wird der Digitalisierungsgedanke nicht explizit herausgearbeitet.

Eine Weiterentwicklung, die sich unmittelbar auf die oben genannten Werkzeuge bezieht und auf Diesntleistungen ausgerichtet ist, stellt die Smart Service Canvas (Pöppelbuss und Durst 2017) für industrienahe Dienstleistungen dar. Sie ist aufgrund ihrer Verständlichkeit und ihrer Analogie zu bekannten Geschäftsmodellen in der Praxis auf die Dienstleistungsentwicklung leicht umsetzbar. Für die Entwicklung und Analyse von PDL kann sie eine wichtige Brücke bilden, deckt aber auch nur Einzelelemente einer wahren Dienstleistungsorientierung ab. So umfasst im konkreten Fall der Smart Service Canvas das beschriebene Ökosystem nur die technische Infrastruktur, nicht aber die arbeitsteilige Einbettung der Kunden in die Dienstleistungserstellung. Auch ist die Entwicklung eines ko-kreativen Value-in-Interaction mit Hilfe der Smart Service Canvas unwahrscheinlich. Denn der Kunde wird prinzipiell als passiver Akteur, ohne die Option einer Kunden-/Nutzerintegration gesehen. Das Wertangebot fokussiert wiederum auf Produkt-Dienstleistungsbündel, bei dem ein smartes Produkt mit Dienstleistungen kombiniert wird. Es gibt eine Unzahl weitere Werkzeuge, die auf wichtige Einzelaspekte referenzieren (siehe hierzu von Engelhardt und Petzold 2019). Eine Methode, die alle PDL relevanten Aspekte umfasst fehlt bisher.

Ein erster, uns bekannter Entwurf für ein Werkzeug für die Bestimmung eines Geschäftsmodells für PDL ist die BeDien Service Canvas (Robra-Bissantz et al. 2020). Diese Canvas wird derzeit im Rahmen des BMBF Projekt „BeDien“ entwickelt, und impliziert die beschriebenen sieben Prinzipien und die damit einhergehende SDLUnternehmensphilosophie.

Ausgangspunkt der BeDien Service Canvas sind die aus der Literatur identifizierten Gestaltungsbereiche der PDL: das zu gestaltende Werteversprechen (Valuein-Use), die Gestaltung der Beziehungen (Value-in-Interaction) zwischen Anbieter und Nutzer, sowie die Konfiguration des zugrundeliegenden Service-Ökosystems mit all seinen Partnern, der Technik und den verfügbaren materiellen und immateriellen Ressourcen. Das gemeinsame Verständnis dieser Gestaltungsbereiche in einem interdisziplinären Team aus Partnern, Kunden und der eigenen Organisation legt die Basis für eine Ko-Kreativität. Darauf aufbauend können die potenziellen Partner im Service-Ökosystem ihre kreative Ideenfindung für die Gestaltung der PDL beginnen. Dabei werden sie über die in den Prinzipien festgehaltenen Entwicklungslinien geleitet und zu neuen Ideen angeregt. So können sie entscheiden, wie und in welchem 
Ausmaß eine Individualisierung von Leistungen, eine Integration von Partnern in der Wertegenerierung, eine Kollaboration mit Partnern, sowie ein digitales Design der Services umgesetzt werden soll.

Die Service Canvas spannt damit einen Raum mit den Achsen „Gestaltungsbereiche“ und „Entwicklungslinien“ auf. Mit Hilfe eines begleitenden Reifegradmodells, mit dem es möglich ist, den Entwicklungsstand der Dienstleistungsgestaltung anhand der genannten Kriterien zu bewerten und zu befördern, können agil die Potenziale für moderne, wertorientierte und erfolgsversprechende PDL entwickelt werden.

Die Service-Canvas rät dann in den Feldern ihrer Matrix (aus den beiden Achsen) beispielweise dazu, eine aktivere Integration des Kunden in die PDL-Innovation sowie in den Wertschöpfungsprozess, zum Beispiel über Maker- oder Do-It-YourselfAnsätze zu erwägen. Sie stellt die Bedeutung der sozialen, kollaborativen Interaktion und der Integration von Wissen der Partner in die Generierung eines Value-inInteraction dar und lässt den PDL-Designer über die Möglichkeiten einer digitalen Schnittstelle zu Partnern nachdenken, in der beispielsweise Chatbots aktiv die Kommunikation übernehmen. Im Dienstleistungs-Ökosystem regt die Reifegradbetrachtung dazu an, nicht allein eigene Werte und Kompetenzen gezielt zu planen, sondern letztere auch agil weiterzuentwickeln. Selbstverständlich ist für die Gestaltung mithilfe der Bedien-Canvas, dass ganzheitlich auf alle potenziell geeigneten Partner geblickt und deren Ressourcen und Kompetenzen gezielt und unter Abwägung der jeweiligen Werte-/Kompetenzenbilanz in das Service-Ökosystem eingebunden sind.

Mit der entsprechenden Geisteshaltung, der Nutzung der Service Canvas, inklusive Reifegradmodell, und in der Anwendung des Service Design Thinking, ergibt sich ein dediziertes Konzept für die Entwicklung von PDL der Zukunft, das versucht, dass die oben beschriebenen sieben Prinzipien (Nutzerzentriertheit, Integration, Kollaboration, Agilität, Ko-Kreativität, Interdisziplinarität, Digitalisierung) durchgehend im Unternehmen reflektiert werden.

Es ist hier darauf hinzuweisen, dass die Bedien Canvas Work-in-Progress darstellt und in den kommenden Jahren anhand von Praxisprojekten evaluiert und angepasst wird.

\section{Zusammenfassender Ausblick}

Dieser Artikel zeigt die aktuelle und zukünftige Bedeutung von PDL für die Gesellschaft und Wirtschaft in einer digitalisierten, Post-Corona-Welt auf. Es zeichnet sich $\mathrm{ab}$, dass PDL in allen sozialen und wirtschaftlichen Bereichen an Bedeutung gewinnen. Dies ergibt sich aus dem Zusammentreffen mehrere Entwicklungen: Zum einen durchdringen Megatrends wie Konnektivität, Sicherheit, Gesundheit und Individualisierung die Dienstleistungsentwicklung und somit PDL. Zum zweiten beschleunigt die Digitalisierung, sowie die Corona-Krise - sich gegenseitig bedingend - den gesellschaftlichen Wandel. Zum dritten hat sich in den vergangenen Jahren, mit der Entwicklung der Digitalisierung der Industrie (Industrie 4.0) ein neues Verständnis der Nutzerzentrierung und der Notwendigkeit der feinen Komposition von Business-Ökosystemen in der Erstellung und für die Erbringung von Dienstleistungen ergeben. Parallel wurde dies in der Wissenschaft mit einer theoretischen Fundierung, 
der SDL, untermauert, die einen Grundstein für eine neue Unternehmensphilosophie legt.

Dieser Artikel hat unter anderem zum Ziel, die Relevanz des Themas PDL für die Wissenschaft und Praxis aufzuzeigen. Nun gilt es gemeinsam geeignete Entwicklungsmethoden für die Komposition PDL mit ganzheitlich gedachten Geschäftsmodellen und Innovationsmethoden aufzusetzen und $\mathrm{zu}$ erproben. Um eine breite Diskussion zu starten, wurden in diesem Beitrag erste Konzepte - die in all ihren Details und Elementen noch lange nicht ausgereift sind und weiterer Überarbeitung bedürfen - vorgestellt. Und nicht nur die einzelnen Konzepte, sondern auch grundlegende wissenschaftliche Erkenntnisse zur Dienstleistungsforschung sowie das unternehmerische Denken müssen sich weiterentwickeln. Die derzeit noch teilweise recht zersplitterten Fachgebiete, die sich mit Dienstleistungen auseinandersetzen, müssen sich zusammenfinden, um den komplexen Ansprüchen von PDL adäquat $\mathrm{zu}$ begegnen. Unternehmen müssen noch viel mehr als heute den Gedanken eine Nutzerorientierung und Nutzerintegration in die Wertschöpfung verstehen und verinnerlichen. Die oben angestellten Überlegungen der reziproken Beeinflussung von Service-Ökosystem und Kundenbedürfnis, mit der Hinzunahme der Spielregeln des „neuen Spiels“ werden entscheidend sein, für die erfolgreichen Post-Corona-PDLSysteme. Die Digitalisierung von PDL ist und bleibt ein spannendes Forschungsfeld.

Danksagung Dieser Beitrag ist Teil des vom Bundesministerium für Bildung und Forschung geförderten Verbundprojekts „BeDien - Begleitforschung Personennahe Dienstleistungen“ (Förderkennzeichen: 02K17A080-81).

Funding Open Access funding provided by Projekt DEAL.

Open Access Dieser Artikel wird unter der Creative Commons Namensnennung 4.0 International Lizenz veröffentlicht, welche die Nutzung, Vervielfältigung, Bearbeitung, Verbreitung und Wiedergabe in jeglichem Medium und Format erlaubt, sofern Sie den/die ursprünglichen Autor(en) und die Quelle ordnungsgemäß nennen, einen Link zur Creative Commons Lizenz beifügen und angeben, ob Änderungen vorgenommen wurden.

Die in diesem Artikel enthaltenen Bilder und sonstiges Drittmaterial unterliegen ebenfalls der genannten Creative Commons Lizenz, sofern sich aus der Abbildungslegende nichts anderes ergibt. Sofern das betreffende Material nicht unter der genannten Creative Commons Lizenz steht und die betreffende Handlung nicht nach gesetzlichen Vorschriften erlaubt ist, ist für die oben aufgeführten Weiterverwendungen des Materials die Einwilligung des jeweiligen Rechteinhabers einzuholen.

Weitere Details zur Lizenz entnehmen Sie bitte der Lizenzinformation auf http://creativecommons.org/ licenses/by/4.0/deed.de.

\section{Literatur}

Bedien.org (2020) Personennahe Dienstleistungen gemeinsam gestalten. https://www.bedien.org/kick-offbedien. Zugegriffen: 29. Juni 2020

Beuth (1998) DIN-Fachbericht 75:1998, Service Engineering - Entwicklungsbegleitende Normung (EBN) für Dienstleistungen. https://www.beuth.de/de/technische-regel/din-fachbericht-75/3725649. Zugegriffen: 29. Juni 2020

Beverungen D, Müller O, Matzner M et al (2017) Conceptualizing smart service systems. Electron Markets 29:1-12

BMBF (2016) Bekanntmachung des Bundesministeriums für Bildung und Forschung; Richtlinie zur Förderung von Maßnahmen für „Personennahe Dienstleistungen“ im Rahmen des Forschungsprogramms 
„Innovationen für die Produktion, Dienstleistung und Arbeit von morgen“. Bundesanzeiger vom 04.10.2016

Böhmann T, Leimeister JM, Möslein K (2014) Service-Systems-Engineering - Ein zukünftiges Forschungsgebiet der Wirtschaftsinformatik. Bus Inf Syst Eng 56(2):83-90

Brenner W, Uebernickel F, Abrell T (2016) Design thinking as mindset, process, and toolbox experiences from research and teaching at the University of St. Gallen. In: Brenner W, Uebernickel F (Hrsg) Design thinking for innovation. Springer, Cham

von Engelhardt S, Petzold S (Hrsg) (2019) Das Geschäftsmodell Toolbook für Digitale Ökosysteme. Campus, Frankfurt, New York, S 65-79

Fischer S, Lattemann C, Redlich B, Guerrero R (2020) Implementation of design thinking to improve organizational agility in an SME. DU 74(2):137-154

Gatterer H (2020) Unsere neue Zukunft: Mit den Megatrends in die Post-Corona-Zeit. https://www. zukunftsinstitut.de/artikel/mit-den-megatrends-in-die-post-corona-zeit/. Zugegriffen: 7. Mai 2020

Geiger M, Robra-Bissantz S, Meyer M (2020) Wie aus digitalen Services Wert entsteht: Interaktionenrichtig gestalten. HMD 57 (2). https://doi.org/10.1365/s40702-020-00611-0

Grönroos C, Voima P (2013) Critical service logic: making sense of value creation and co-creation. J Acad Mark Sci 41(2):133-150

Hipp C (2013) Innovationsprozesse im Dienstleistungssektor: Eine theoretisch und empirisch basierte Innovationstypologie Bd. 40. Springer, Heidelberg

Hofmann J (2020) Incentivierung von Prosumern auf einem elektronischen Marktplatz für personennahe Dienstleistungen. HMD 57(4). https://doi.org/10.1365/s40702-020-00631-w

Johansson-Sköldberg U, Woodilla J, Çetinkaya M (2013) Design thinking: past, present and possible futures. Creat Innov Manag 22(2):121-146

Lattemann C, Robra-Bissantz S (2006) Customer governance - IC based concepts for a successful customer integration. In: Hannula M, Järvelin AM, Seppä M (Hrsg) Frontiers of e-Business Research FeBR2005.

Lattemann C, Robra-Bissantz S, Fischer S, Ahmad R (2019) Personennahe Dienstleistungen in der digitalen Transformation. IM+io 2019(1):74-77

Matthews J, Wrigley C (2011) Design and design thinking in business and management education and development. In: Proceedings of the 25th Annual Australian and New Zealand Academy of Management Conference, S 1-19

Osterwalder A, Pigneur Y, Tucci CL (2005) Clarifying business models: origins, present, and future of the concept. Commun Assoc Inf Syst 16(16):1-25

Osterwalder A, Pigneur Y, Bernarda G, Smith A, Papadakos T (2014) Value proposition design: how to create products and services customers want. Wiley, London

Pöppelbuß J, Durst C (2017) Smart Service Canvas - Ein Werkzeug zur strukturierten Beschreibung und Entwicklung von Smart-Service-Geschäftsmodellen. In: Bruhn M, Hadwich K (Hrsg) Dienstleistungen 4.0. Gabler, Wiesbaden, S 92-111

Redlich B, Becker F, Fischer S et al (2019) Das DETHIS-Verfahren. In: Stich V, Schumann J, Beverungen D et al (Hrsg) Digitale Dienstleistungsinnovationen. Springer Vieweg, Berlin, Heidelberg

Robra-Bissantz S (2018) Entwicklung von innovativen Services in der Digitalen Transformation. In: Bruhn M, Hadwich K (Hrsg) Service business development. Springer Gabler, Wiesbaden, S 261-288

Robra-Bissantz S, Lattemann C (Hrsg) (2019) Digital Customer Experience - Mit digitalen Diensten Kunden gewinnen und halten. Springer Vieweg, Berlin, Heidelberg

Robra-Bissantz S, Lattemann C, Guerrero R et al (2020) Der Mensch als Teil der Innovation - Eine ,Service Canvas“ als anwendungsorientierter Bezugsrahmen. In: Bruhns M, Hadwich K (Hrsg) Dienstleistungsmanagement 2020 „Automatisierung und Personalisierung von Dienstleistungen“. Springer Gabler, Wiesbaden (forthcoming)

Stieglitz S, Lattemann C, Robra-Bissantz S et al (Hrsg) (2017) Gamification: using game elements in serious contexts. Springer, Cham

Vargo SL, Lusch RF (2011) It's all B2B ... and beyond: toward a systems perspective of the market. Ind Mark Manag 40(2):181-187

Vargo SL, Lusch RF (2016) Institutions and axioms: an extension and update of service-dominant logic. J Acad Mark Sci 44(1):5-23

Wieland H, Polese F, Vargo SL, Lusch RF (2012) Toward a service (eco)-systems perspective on value creation. Int J Serv Sci Manag Eng Technol 3(3):12-25

Zollenkop M, Lässig R (2017) Digitalisierung im Industriegütergeschäft. In: Schallmo D et al (Hrsg) Digitale Transformation von Geschäftsmodellen. Springer, Wiesbaden, S 59-95

Zukunftsinstitut (2020) Megatrends. https://www.zukunftsinstitut.de/dossier/megatrends/. Zugegriffen: 29. Juni 2020 